\title{
Fixed-Complexity Piecewise Ellipsoidal Representation of the Continual Reachability Set Based on Ellipsoidal Techniques
}

\author{
Shahab Kaynama, Meeko Oishi, Ian M. Mitchell, and Guy A. Dumont
}

\begin{abstract}
In a previous paper we showed how the continual reachability set can be numerically computed using efficient maximal reachability tools. The resulting set is in general arbitrarily shaped and in practice possibly non-convex. Here, we present a fixed-complexity piecewise ellipsoidal under-approximation of the continual reachability set computed using ellipsoidal techniques. This provides a simple approximation of an otherwise relatively complicated set that can be used when a closed-form representation is needed. We demonstrate the results on a problem of control of anesthesia.
\end{abstract}

\section{INTRODUCTION}

Mathematical guarantees of safety and performance are crucial components of robust-by-design cyberphysical systems. Reachability analysis has typically been used to provide safety certificates (e.g. for collision avoidance or flight envelope protection) for constrained dynamical systems despite bounded control authority [1]-[5]. It has also been used to synthesize bounded input policies that can steer the system to a given target [6], [7]. Reachability analysis identifies the set of states backward (forward) reachable by a constrained dynamical system from a target (initial) set of states.

In many applications, however, the state constraints may be temporarily relaxed in favor of improved performance. Motivated by a problem of guaranteed performance and safety in control of anesthesia, we introduced the continual reachability set [8] which can be used to provide guarantees of performance in reachability analysis. The continual reachability set is the set of states that can reach the target at any given time within the finite horizon. Initiating the system from this set provides additional flexibility to a supervisory controller to choose a policy that optimizes a trade-off between the desired timeto-reach the target and the input effort (or other performance indices) required to drive the state to that target.

It is well known [9] that the required optimal control laws that keep the system within a given target set are generally discontinuous or even bang-bang. In the anesthesia setting, such optimal control laws (drug infusion rates) may not be physiologically suitable for the current conditions of a patient in the operating room. Less aggressive and more patient oriented control policies may be warranted. In such scenarios,

Research supported by NSERC Discovery Grant \#327387 and \#298211, NSERC Collab. Health Research Project \#CHRPJ-350866-08, and the Institute for Computing, Information and Cognitive Systems (ICICS) at UBC.

S. Kaynama (cor. author) and G.A. Dumont are with the Dept. of Electrical and Computer Engineering, University of British Columbia, Vancouver, BC, Canada $\{k a y n a m a$, guyd $\}$ eece.ubc.ca

M. Oishi is with the Dept. of Electrical and Computer Engineering, University of New Mexico, Albuquerque, NM, USA oishi@unm. edu

I.M. Mitchell is with the Dept. of Computer Science, University of British Columbia, Vancouver, BC, Canada mitchell@cs. ubc. ca one may temporarily allow the relaxation of the target therapeutic bounds, and in return provide the anesthesiologist (or a closed-loop controller) with additional degrees of freedom in choosing a better suited drug infusion while simultaneously ensuring that the target clinical effect is reached at a given time in the future.

A variety of approaches to controlling depth of anesthesia have been proposed [10]-[15] to improve patient recovery, lessen anesthetic drug usage, and reduce time spent at drug saturation levels. The continual reachability set provides a guarantee of performance, in that for any initial state in the set, the desired clinical effect can be reached at arbitrary times. This may be particularly useful in scenarios in which one wishes to minimize the total administered drug, or to achieve a desired depth of anesthesia arbitrarily fast.

Building upon our previous work in formulating the continual reachability set, our main contribution in this paper is to provide a technique that can be used to approximate this geometrically complicated set when a simple closed-form representation of fixed-complexity is needed. We then apply this technique to our problem of control of anesthesia. We note that while the approximation of the continual reachability set is motivated by a highly specific problem in closed-loop anesthesia delivery, our method is general, and may also be of use in other engineering domains for which safety constraints may be temporarily relaxed in favor of performance goals.

Section II formulates the problem of simplifying the representation of the continual reachability set. Section III presents a solution based on ellipsoids. Section IV demonstrates the results on the anesthesia problem. Concluding remarks are provided in Section $\mathrm{V}$.

\section{Problem Formulation}

Consider the discrete-time linear time-invariant system

$$
x(t+1)=A x(t)+B u(t), \quad x(0)=x_{0}, \quad t \in \mathbb{Z}^{+}
$$

with state space $\mathcal{X}:=\mathbb{R}^{n}$, state vector $x(t) \in \mathcal{X}$, and input $u(t) \in \mathcal{U}$ where $\mathcal{U}$ is a compact convex subset of $\mathbb{R}^{m}$. Denote by $\mathscr{U}_{[0, t]}$ the set of functions $u(\cdot)$ from $[0, t]$ to $\mathcal{U}$. With an arbitrary finite time horizon $\tau \in \mathbb{Z}^{+}, \tau>0$, for every $t \in[0, \tau], x_{0} \in \mathcal{X}$, and $u(\cdot) \in \mathscr{U}_{[0, t]}$, there exists a unique trajectory $\xi_{x_{0}, u(\cdot)}:[0, t] \rightarrow \mathcal{X}$ that satisfies the initial condition $\xi_{x_{0}, u(\cdot)}(0)=x_{0}$ and the difference equation (1).

For a nonempty state constraint set $\mathcal{K} \subseteq \mathcal{X}$ we examine the following backward constructs:

Definition 1 (Maximal Reachability Set). The maximal reachability set at time $t \in[0, \tau]$ is the set of initial states for which 
there exists an input $u(\cdot)$ such that the trajectories emanating from those states reach $\mathcal{K}$ exactly at time $t:$

$$
\operatorname{Reach}_{t}^{\sharp}(\mathcal{K}):=\left\{x_{0} \in \mathcal{X} \mid \exists u(\cdot) \in \mathscr{U}_{[0, t]}, \xi_{x_{0}, u(\cdot)}(t) \in \mathcal{K}\right\} .
$$

Definition 2 (Continual Reachability Set). The continual reachability set defined over the time horizon $[0, \tau]$ is the set of initial states in $\mathcal{K}$ for which, for any given time $t \in[0, \tau]$, there exists a $u(\cdot)$ such that the trajectories emanating from those states reach $\mathcal{K}$ at $t$ :

$$
\begin{array}{r}
\operatorname{Reach}_{[0, \tau]}^{\gamma}(\mathcal{K}):=\left\{x_{0} \in \mathcal{X} \mid \forall t \in[0, \tau], \exists u(\cdot) \in \mathscr{U}_{[0, t]},\right. \\
\left.\xi_{x_{0}, u(\cdot)}(t) \in \mathcal{K}\right\} .
\end{array}
$$

In [8] we showed that

$$
\operatorname{Reach}_{[0, \tau]}^{\gamma}(\mathcal{K})=\bigcap_{t \in[0, \tau]} \operatorname{Reach}_{t}^{\sharp}(\mathcal{K}) .
$$

Thus, when $\mathcal{K}$ and $\mathcal{U}$ are (or can be reasonably underapproximated by) compact nonempty ellipsoids, ellipsoidal techniques [16] implemented in the Ellipsoidal Toolbox (ET) [17] can be used to compute a compact approximation of $\operatorname{Reach}_{[0, \tau]}^{\gamma}(\mathcal{K})$.

Definition 3 (Ellipsoid). An ellipsoid in $\mathbb{R}^{n}$ is defined as

$$
\mathcal{E}(q, Q):=\left\{x \in \mathbb{R}^{n} \mid\left\langle(x-q), Q^{-1}(x-q)\right\rangle \leq 1\right\}
$$

with center $q \in \mathbb{R}^{n}$ and shape matrix $\mathbb{R}^{n \times n} \ni Q=Q^{T} \succ 0$.

Let $\mathcal{K}_{\downarrow \varepsilon}:=\mathcal{E}\left(x_{\tau}, X_{\tau}\right) \subset \mathcal{X}$ be a nonempty ellipsoidal under-approximation of $\mathcal{K}$ and assume $\mathcal{U}=\mathcal{E}(p, P) \subset \mathbb{R}^{m}$. With $\mathcal{L}:=\left\{l \in \mathbb{R}^{n} \mid\langle l, l\rangle=1\right\}$, the following holds:

$$
\begin{aligned}
\operatorname{Reach}_{[0, \tau]}^{\gamma}(\mathcal{K}) & \supseteq \operatorname{Reach}_{[0, \tau]}^{\gamma}\left(\mathcal{K}_{\downarrow \varepsilon}\right) \\
& =\bigcap_{t \in[0, \tau]}\left(\bigcup_{\ell_{\tau} \in \mathcal{L}} \mathcal{E}\left(x^{*}(t), X_{\ell}^{-}(t)\right)\right),
\end{aligned}
$$

where $x^{*}(t)$ and $X_{\ell}^{-}(t)$ are the center vector and the shape matrix of the internal approximating ellipsoid at time $t$ that is tangent to $\operatorname{Reach}_{t}^{\sharp}\left(\mathcal{K}_{\downarrow \varepsilon}\right)$ in the direction $\ell(t)$. For a fixed $\ell(\tau)=\ell_{\tau} \in \mathcal{L}$, the direction $\ell(t)$ is obtained from the adjoint equation $\ell(t+1)=\left(A^{T}\right)^{-1} \ell(t)$. The center $x^{*}(t)$ (with $x^{*}(\tau)=x_{\tau}$ ) and the shape matrix $X_{\ell}^{-}(t)$ (with $X_{\ell}^{-}(\tau)=X_{\tau}$ ) are determined from recurrence relations described in [18].

In practice, only a finite number of directions can be used for maximal reachability set computations. Let $\mathcal{J}$ be a finite subset of $\mathcal{L}$. We also showed in [8] that

$$
\begin{aligned}
\operatorname{Reach}_{[0, \tau]}^{\gamma}(\mathcal{K}) & \supseteq \operatorname{Reach}_{[0, \tau]}^{\gamma}\left(\mathcal{K}_{\downarrow \varepsilon}\right) \\
& \supseteq \bigcap_{t \in[0, \tau]}\left(\bigcup_{\ell_{\tau} \in \mathcal{J}} \mathcal{E}\left(x^{*}(t), X_{\ell}^{-}(t)\right)\right),
\end{aligned}
$$

implying that the continual reachability set is correctly underapproximated. A direct consequence of (5) is that

$$
\begin{aligned}
& \operatorname{Reach}_{[0, \tau]}^{\gamma}(\mathcal{K}) \supseteq \operatorname{Reach}_{[0, \tau]}^{\gamma}\left(\mathcal{K}_{\downarrow \varepsilon}\right) \supseteq \\
&\left\{x \in \mathcal{X} \mid \max _{t \in[0, \tau] \ell_{\tau} \in \mathcal{J}}\left\langle\left(x-x^{*}(t)\right),\right.\right. \\
&\left.\left.\left(X_{\ell}^{-}(t)\right)^{-1}\left(x-x^{*}(t)\right)\right\rangle \leq 1\right\} .
\end{aligned}
$$

Notice that while the ellipsoidal-based representation in (4) is convex (for a linear system with convex constraints the continual reachability set is also convex [8]), the under-approximation in (5) may not be: The union $\bigcup_{\ell_{\tau} \in \mathcal{J}} \mathcal{E}\left(x^{*}(t), X_{\ell}^{-}(t)\right)$ at any $t \in[0, \tau]$ is an arbitrarily shaped, possibly non-convex set, and so is the intersection of these sets over all $t$.

Moreover, while from a numerical standpoint it is easy to determine whether a given point in the state space belongs to $\operatorname{Reach}_{[0, \tau]}^{\gamma}(\mathcal{K})$ by evaluating (6) at that point, there are instances where having a simple closed-form representation of (5) (or equivalently (6)) is desirable. One such instance is when finding the (generalized) gradient at the boundary points of $\operatorname{Reach}_{[0, \tau]}^{\gamma}(\mathcal{K})$ or its under-approximation, which might prove helpful for synthesis of the continual reachability control laws.

Problem 1. Formulate a simple, closed-form representation of the continual reachability set based on the inclusion in (5).

\section{PieCEWISE Ellipsoidal REPRESENTATION}

We address Problem 1 using a fixed-complexity piecewise ellipsoidal approach. A piecewise ellipsoidal set is the union of a finite number of ellipsoids.

\section{Lemma 1.}

$$
\begin{aligned}
& \bigcup_{\ell_{\tau} \in \mathcal{J}}\left(\bigcap_{t \in[0, \tau]} \mathcal{E}\left(x^{*}(t), X_{\ell}^{-}(t)\right)\right) \\
& \quad \subseteq \bigcap_{t \in[0, \tau]}\left(\bigcup_{\ell_{\tau} \in \mathcal{J}} \mathcal{E}\left(x^{*}(t), X_{\ell}^{-}(t)\right)\right) \subseteq \operatorname{Reach}_{[0, \tau]}^{\gamma}\left(\mathcal{K}_{\downarrow \varepsilon}\right) .
\end{aligned}
$$

Proof: $x \in \bigcup_{\ell_{\tau} \in \mathcal{J}}\left(\bigcap_{t \in[0, \tau]} \mathcal{E}\left(x^{*}(t), X_{\ell}^{-}(t)\right)\right) \Longleftrightarrow$ $\left(\exists \ell_{\tau} \in \mathcal{J}\right)(\forall t \quad \in[0, \tau]) x \in \mathcal{E}\left(x^{*}(t), X_{\ell}^{-}(t)\right) \Longrightarrow$ $(\forall t \in[0, \tau])\left(\exists \ell_{\tau} \in \mathcal{J}\right) x \in \mathcal{E}\left(x^{*}(t), X_{\ell}^{-}(t)\right) \Longleftrightarrow x \in$ $\bigcap_{t \in[0, \tau]}\left(\bigcup_{\ell_{\tau} \in \mathcal{J}} \mathcal{E}\left(x^{*}(t), X_{\ell}^{-}(t)\right)\right)$.

Lemma 1 asserts that the intersection of many arbitrarilyshaped, possibly non-convex sets (each formed by the union of $|\mathcal{J}|$ ellipsoids) which is hard to compute can be underapproximated by the union of $|\mathcal{J}|$ arbitrarily-shaped convex sets (each formed by the intersection of many ellipsoids) which, as we shall see, is easy to approximate.

Consider the term $\bigcap_{t \in[0, \tau]} \mathcal{E}\left(x^{*}(t), X_{\ell}^{-}(t)\right)$ in Lemma 1. While the intersection of any two or more ellipsoids in general is not an ellipsoid, this intersection can be under-approximated by a maximum volume inscribed ellipsoid [19] (a readily available feature in ET): With $\tau$ time steps, for a given direction $\ell_{\tau} \in \mathcal{J}$ and assuming $\operatorname{int}\left(\bigcap_{t \in[0, \tau]} \mathcal{E}\left(x^{*}(t), X_{\ell}^{-}(t)\right)\right) \neq \emptyset$, the following semidefinite program [19] is solved.

$$
\begin{gathered}
\underset{Y_{\ell_{\tau}} \in \mathbb{R}^{n \times n}, y_{\ell_{\tau}} \in \mathbb{R}^{n}, \lambda_{i} \in \mathbb{R}}{\operatorname{minimize}} \log \operatorname{det} Y_{\ell_{\tau}}^{-1} \\
\text { subject to } \lambda_{i}>0 \\
{\left[\begin{array}{ccc}
1-\lambda_{i} & 0 & \left(y_{\ell_{\tau}}-x^{*}(i)\right)^{T} \\
0 & \lambda_{i} I & Y_{\ell_{\tau}} \\
y_{\ell_{\tau}}-x^{*}(i) & Y_{\ell_{\tau}} & X_{\ell}^{-}(i)
\end{array}\right]} \\
\quad i=0, \ldots, \tau .
\end{gathered}
$$


The resulting ellipsoid $\mathcal{E}\left(y_{\ell_{\tau}}, Y_{\ell_{\tau}}^{T} Y_{\ell_{\tau}}\right)$ is the maximum volume inscribed ellipsoid corresponding to $\ell_{\tau}$.

\section{Proposition 1.}

$$
\bigcup_{\ell_{\tau} \in \mathcal{J}} \mathcal{E}\left(y_{\ell_{\tau}}, Y_{\ell_{\tau}}^{T} Y_{\ell_{\tau}}\right) \subseteq \operatorname{Reach}_{[0, \tau]}^{\gamma}\left(\mathcal{K}_{\downarrow \varepsilon}\right) .
$$

Proof: $\mathcal{E}\left(y_{\ell_{\tau}}, Y_{\ell_{\tau}}^{T} Y_{\ell_{\tau}}\right) \subseteq \bigcap_{t \in[0, \tau]} \mathcal{E}\left(x^{*}(t), X_{\ell}^{-}(t)\right)$ for every $\ell_{\tau} \in \mathcal{J}$. Thus, $\bigcup_{\ell_{\tau} \in \mathcal{J}} \mathcal{E}\left(y_{\ell_{\tau}}, Y_{\ell_{\tau}}^{T} Y_{\ell_{\tau}}\right) \subseteq$ $\bigcup_{\ell_{\tau} \in \mathcal{J}}\left(\bigcap_{t \in[0, \tau]} \mathcal{E}\left(x^{*}(t), X_{\ell}^{-}(t)\right)\right)$. Therefore, by Lemma 1, $\bigcup_{\ell_{\tau} \in \mathcal{J}} \mathcal{E}\left(y_{\ell_{\tau}}, Y_{\ell_{\tau}}^{T} Y_{\ell_{\tau}}\right) \subseteq \operatorname{Reach}_{[0, \tau]}^{\gamma}\left(\mathcal{K}_{\downarrow \varepsilon}\right)$.

Remark 1. The left-hand-side in (8) is a piecewise ellipsoidal approximation of the continual reachability set. As a result, instead of one arbitrarily-shaped set in (5) (formed by taking the intersection of union of ellipsoids), we now have an underapproximation of the continual reachability set expressed in terms of $|\mathcal{J}|=\left|\left\{\mathcal{E}\left(y_{\ell_{\tau}}, Y_{\ell_{\tau}}^{T} Y_{\ell_{\tau}}\right)\right\}_{\ell_{\tau} \in \mathcal{J}}\right|$ ellipsoids.

\section{A. Maximum k-Connected Ellipsoidal Representation}

To further simplify the above representation, we introduce a technique that maximally spans the volume of the set $\bigcup_{\ell_{\tau} \in \mathcal{J}} \mathcal{E}\left(y_{\ell_{\tau}}, Y_{\ell_{\tau}}^{T} Y_{\ell_{\tau}}\right)$ subject to using at most $k \leq|\mathcal{J}|$ of its subset ellipsoids. Note that we still require an underapproximation since an over-approximation of the above union (or any of its subsets) does not necessarily guarantee the underapproximation of the continual reachability set.

For a given $k \in \mathbb{N}$, we seek $\mathcal{I} \subseteq \mathcal{J}$ such that $|\mathcal{I}| \leq k$ and $\left|\bigcup_{\ell_{\tau} \in \mathcal{I}} \mathcal{E}\left(y_{\ell_{\tau}}, Y_{\ell_{\tau}}^{T} Y_{\ell_{\tau}}\right)\right|$ is maximized, or equivalently, $d_{H}\left(\bigcup_{\ell_{\tau} \in \mathcal{I}} \mathcal{E}\left(y_{\ell_{\tau}}, Y_{\ell_{\tau}}^{T} Y_{\ell_{\tau}}\right), \bigcup_{\ell_{\tau} \in \mathcal{J}} \mathcal{E}\left(y_{\ell_{\tau}}, Y_{\ell_{\tau}}^{T} Y_{\ell_{\tau}}\right)\right)$ with $d_{H}$ denoting the Hausdorff distance, is minimized. In addition, we also require that the subset $\mathcal{I}$ is chosen such that

$$
\begin{aligned}
& \forall \widetilde{\mathcal{I}} \subset \mathcal{I}, \widetilde{\mathcal{I}} \neq \emptyset, \exists \widehat{\mathcal{I}} \subset \mathcal{I}, \widetilde{\mathcal{I}} \cap \widehat{\mathcal{I}}=\emptyset, \exists \tilde{\ell}_{\tau} \in \widetilde{\mathcal{I}}, \\
& \quad \exists \hat{\ell}_{\tau} \in \widehat{\mathcal{I}}, \mathcal{E}\left(y_{\hat{\ell}_{\tau}}, Y_{\hat{\ell}_{\tau}}^{T} Y_{\hat{\ell}_{\tau}}\right) \cap \mathcal{E}\left(y_{\tilde{\ell}_{\tau}}, Y_{\tilde{\ell}_{\tau}}^{T} Y_{\tilde{\ell}_{\tau}}\right) \neq \emptyset .
\end{aligned}
$$

This condition ensures that the resulting piecewise ellipsoidal set is a connected set. Doing so provides a trajectory with the possibility of traversing anywhere within the set when needed.

We refer to this problem as the Maximum $k$-Connected Ellipsoidal Representation ( $\mathrm{kCER}$ ) of the continual reachability set. As it turns out, kCER is a variation of the maximum coverage problem [20]. For simplicity, let $\left\{\mathcal{E}\left(y_{\ell_{\tau}}, Y_{\ell_{\mathcal{I}}}^{T} Y_{\ell_{\tau}}\right)\right\}_{\ell_{\tau} \in \mathcal{J}}$ be denoted by an indexed family of sets $\left\{\mathcal{E}_{i}\right\}_{i=1}^{|\mathcal{J}|}$. We formulate kCER as a binary integer program while tackling the connectedness requirement (9) via a graph-theoretic approach:

$$
\begin{array}{ll}
\underset{z_{1}, \ldots, z_{|\mathcal{J}|}}{\operatorname{maximize}} & \sum_{i=1}^{|\mathcal{J}|}\left(c_{i}-d_{i}\right) z_{i} \\
\text { subject to } & c_{i}=\operatorname{vol}\left(\mathcal{E}_{i}\right) \\
& d_{i}=\sum_{j=1}^{|\mathcal{J}|} \frac{\operatorname{vol}\left(\mathcal{E}_{i} \cap \mathcal{E}_{j}\right)}{\operatorname{vol}\left(\mathcal{E}_{i}\right)} \\
& \sum_{i=1}^{|\mathcal{J}|} z_{i} \leq k
\end{array}
$$

$$
\begin{aligned}
& \sum_{i=1}^{|\mathcal{J}|} \sum_{j=1}^{|\mathcal{J}|} z_{i}\left(\gamma_{i j}^{(k-1)}-1\right) z_{j}=0 \\
& z_{i} \in\{0,1\}, \quad i=1, \ldots,|\mathcal{J}| .
\end{aligned}
$$

Here, $\operatorname{vol}\left(\mathcal{E}_{i}\right)$ is the volume of the $i$-th ellipsoid which can be obtained from the determinant of its shape matrix. In addition, $\operatorname{vol}\left(\mathcal{E}_{i} \cap \mathcal{E}_{j}\right)$ is assumed to be directly proportional to the volume of the maximum volume inscribed ellipsoid in the intersection of $\mathcal{E}_{i}$ and $\mathcal{E}_{j}$. Moreover, $\operatorname{vol}\left(\mathcal{E}_{i} \cap \mathcal{E}_{j}\right)=0$ if $\mathcal{E}_{i} \cap \mathcal{E}_{j}=\emptyset$ and $\operatorname{vol}\left(\mathcal{E}_{i} \cap \mathcal{E}_{j}\right)=\operatorname{vol}\left(\mathcal{E}_{i}\right)$ if $\mathcal{E}_{i} \subseteq \mathcal{E}_{j}$. Notice that $\operatorname{vol}\left(\mathcal{E}_{i}\right) \propto\left|\mathcal{E}_{i}\right|$. Therefore, the optimizer selects those ellipsoids (by setting their corresponding binary variable to 1 ) that have the largest cardinality, while favoring those that are least "covered" by their neighboring ellipsoids; (10a), (10b), and (10c). The constraint (10d) ensures that the cardinality of the set $\mathcal{I}$ (i.e. the number of chosen ellipsoids) is at most $k$. The equality constraint (10e), which ensures that the connectedness requirement (9) is satisfied, as well as the constant $\gamma_{i j}^{(k-1)}$ are related to properties of the graph representation of $\left\{\mathcal{E}_{i}\right\}_{i=1}^{|\mathcal{J}|}$. This is discussed next.

1) Description of Constraint (10e): We begin with a few basic definitions: A graph $\mathcal{G}$ is an ordered pair $(\mathscr{V}, \mathscr{E})$ consisting of a set of vertices $\mathscr{V}:=\left\{v_{1}, \ldots, v_{n}\right\}$ and a set of edges $\mathscr{E} \subseteq\left\{\left(v_{i}, v_{j}\right) \mid v_{i}, v_{j} \in \mathscr{V}\right\}$ such that each edge of $\mathcal{G}$ joins an unordered (not necessarily distinct) pair of its vertices. A graph is undirected if the edges have no orientation; $\left(v_{i}, v_{j}\right) \in \mathscr{E} \Leftrightarrow\left(v_{j}, v_{i}\right) \in \mathscr{E}$. A loop (or, selfloop) is an edge between a vertex and itself. A path is a subgraph $(\widetilde{\mathcal{V}}, \widetilde{\mathscr{E}})$ such that for $\widetilde{\mathcal{V}}:=\left\{v_{1}, v_{2}, \ldots, v_{k}\right\}$ we have $\widetilde{\mathscr{E}}:=\left\{\left(v_{1}, v_{2}\right),\left(v_{2}, v_{3}\right), \ldots,\left(v_{k-1}, v_{k}\right)\right\}$. A walk of length $k$ in $\mathcal{G}$ is an alternating sequence $v_{1} e_{1} v_{2} e_{2} \ldots e_{k-1} v_{k}$ of vertices and edges such that $e_{i}:=\left(v_{i}, v_{i+1}\right)$ for all $i<k$. The order $|\mathcal{G}|$ of a graph $\mathcal{G}$ is the number of its vertices $|\mathscr{V}|$. A graph $\mathcal{G}$ is connected if any two of its vertices are joined by a path in $\mathcal{G}$. A maximal connected subgraph of $\mathcal{G}$ is called a connected component of $\mathcal{G}$. Two vertices $v_{i}, v_{j} \in \mathscr{V}$ are adjacent if $\left(v_{i}, v_{j}\right) \in \mathscr{E}$. The adjacency matrix $A_{\mathcal{G}}:=\left[a_{i j}^{(1)}\right]_{n \times n}$, $n=|\mathscr{V}|$, of $\mathcal{G}$ is defined by

$$
a_{i j}^{(1)}:= \begin{cases}1 & \text { if }\left(v_{i}, v_{j}\right) \in \mathscr{E} \\ 0 & \text { o.w. }\end{cases}
$$

Lemma 2 ([21]). The matrix $\left(A_{\mathcal{G}}\right)^{k}=\left[a_{i j}^{(k)}\right]_{n \times n}$ contains the number $a_{i j}^{(k)}$ of walks of length $k$ from $v_{i}$ to $v_{j}$ in $\mathcal{G}$.

Lemma 3. The matrix polynomial

$$
\psi_{k}\left(A_{\mathcal{G}}\right):=\sum_{\sigma=1}^{k}\left(A_{\mathcal{G}}\right)^{\sigma}=\left[\widetilde{a}_{i j}^{(k)}\right]_{n \times n}
$$

contains the number $\widetilde{a}_{i j}^{(k)}$ of walks of length $l=\left(\bigvee_{\sigma=1}^{k} \sigma\right)$ from $v_{i}$ to $v_{j}$ in $\mathcal{G}$.

Proof: The proof is by a simple induction on $k$. For $k=1, \psi_{1}\left(A_{\mathcal{G}}\right)=A_{\mathcal{G}}$ is the adjacency matrix. The results follows from the definition of $A_{\mathcal{G}}$ since there are $a_{i j}^{(1)}$ walks 

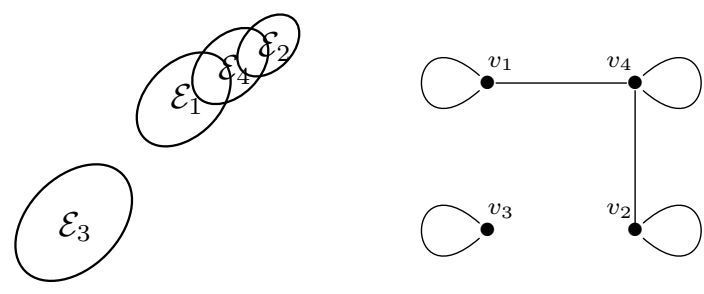

Fig. 1. A piecewise ellipsoidal set (left) and its corresponding graph (right). Each vertex $v_{i}$ of the graph corresponds to an ellipsoid $\mathcal{E}_{i}$ in the piecewise ellipsoidal set (where the sets are arbitrarily labeled). An edge between adjacent vertices indicates a nonempty intersection between their respective ellipsoids.

of length one from $v_{i}$ to $v_{j}$ iff there exists $a_{i j}^{(1)}$ edges between them. Suppose $\psi_{k-1}\left(A_{\mathcal{G}}\right)$ contains the number $\widetilde{a}_{i j}^{(k-1)}$ of walks of length $\left(\bigvee_{\sigma=1}^{k-1} \sigma\right)$ from $v_{i}$ to $v_{j}$. Then, $\psi_{k}\left(A_{\mathcal{G}}\right)=$ $\left(A_{\mathcal{G}}\right)^{k}+\psi_{k-1}\left(A_{\mathcal{G}}\right)$ contains (by Lemma 2 and the induction hypothesis) the number $a_{i j}^{(k)}+\widetilde{a}_{i j}^{(k-1)}=\widetilde{a}_{i j}^{(k)}$ of walks of length $l=\left(k \vee\left(\bigvee_{\sigma=1}^{k-1} \sigma\right)\right)=\left(\bigvee_{\sigma=1}^{k} \sigma\right)$ from $v_{i}$ to $v_{j}$.

Lemma 3 asserts that, from any vertex $v_{i}$ in $\mathcal{G}$, the vertex $v_{j}$ is $\widetilde{a}_{i j}^{(k)}$-times reachable (meaning there exists $\widetilde{a}_{i j}^{(k)}$ distinct paths from $v_{i}$ to $v_{j}$ ) in at most $k$ steps.

Define a Boolean matrix for $A_{\mathcal{G}}$ as

$$
\Gamma\left(A_{\mathcal{G}}\right):=\left[\gamma_{i j}^{(1)}\right]_{n \times n}
$$

such that $\gamma_{i j}^{(1)}=0$ if $a_{i j}^{(1)}=0$ and $\gamma_{i j}^{(1)}=1$ if $a_{i j}^{(1)} \neq 0$. Then a non-zero entry of $\Gamma\left(\psi_{k}\left(A_{\mathcal{G}}\right)\right)$, denoted by $\gamma_{i j}^{(k)}$, indicates that in $\mathcal{G}$, the vertex $v_{j}$ is reachable from $v_{i}$ in at most $k$ steps.

To address the connectedness requirement (9) in KCER, we treat each ellipsoid $\mathcal{E}_{i}$ as a vertex of an undirected graph whose edges represent nonempty intersections between these sets. (Note that determining if any two ellipsoids intersect is a simple convex optimization problem [19].) For example, Fig. 1 shows a piecewise ellipsoidal set and its corresponding graph representation. Self-loops are employed to accommodate the case in which no two ellipsoids intersect, resulting in a graph that consists of isolated vertices.

Let the piecewise ellipsoidal set $\mathcal{E}_{p w}:=\left\{\mathcal{E}_{i}\right\}_{i=1}^{N}$ be represented by an undirected graph $\mathcal{G}=(\mathscr{V}, \mathscr{E})$ such that $v_{i} \in \mathscr{V}$ corresponds to $\mathcal{E}_{i} \in \mathcal{E}_{p w}$ and $\left(v_{i}, v_{j}\right) \in \mathscr{E}$ if and only if $\mathcal{E}_{i} \cap \mathcal{E}_{j} \neq \emptyset$. Let $\left(v_{i}, v_{i}\right) \in \mathscr{E}, \forall v_{i} \in \mathscr{V}$. Let $A_{\mathcal{G}} \in \mathbb{R}^{N \times N}$ be the adjacency matrix of $\mathcal{G}$. Consider a binary vector $z=\left[z_{1} \cdots z_{N}\right]^{T} \in\{0,1\}^{N}$ associated with the set of vertices $\mathscr{V}$. We say that a vertex $v_{i} \in \mathscr{V}$ is selected if and only if its corresponding binary variable is enabled, i.e. $z_{i}=1$. A subgraph $\widetilde{\mathcal{G}}=(\widetilde{\mathscr{V}}, \widetilde{\mathscr{E}}) \subseteq \mathcal{G}$ is selected if and only if $z_{i}=1$ for all $v_{i} \in \widetilde{\mathscr{V}}$ and $z_{i}=0$ for all $v_{i} \in \mathscr{V} \backslash \widetilde{\mathscr{V}}$.

Proposition 2 (Connectedness Condition). Suppose that, for a given $k \leq N$, a subgraph $\widetilde{\mathcal{G}} \subseteq \mathcal{G}$ is to be selected such that $|\widetilde{\mathcal{G}}|=k$. Then,

$$
\left\langle z,\left(\Gamma\left(\psi_{k-1}\left(A_{\mathcal{G}}\right)\right)-\mathbf{1}\right) z\right\rangle=0
$$

or equivalently,

$$
\sum_{i=1}^{N} \sum_{j=1}^{N} z_{i}\left(\gamma_{i j}^{(k-1)}-1\right) z_{j}=0
$$

if and only if $\widetilde{\mathcal{G}}$ is connected. ${ }^{1}$ Furthermore, this equality holds for every selected subgraph of $\widetilde{\mathcal{G}}$, with order less than $k$, that is connected.

Proof: $(\Rightarrow$ ): Assume (14) holds for $\widetilde{\mathcal{G}} \subseteq \mathcal{G} .|\widetilde{\mathcal{G}}|=k$ means exactly $k$ number of $z_{i}$ 's are 1 . Take any two vertices $v_{i}$ and $v_{j}$. Suppose $v_{i}$ and $v_{j}$ are selected. Thus, $z_{i}=1$ and $z_{j}=1$. Therefore, (14) can only hold when $\gamma_{i j}^{(k-1)}=1$, meaning that $v_{i}$ and $v_{j}$ are joined by a path of length at most $k-1$ edges.

$(\Leftarrow)$ : (By contrapositive.) Assume (14) does not hold for some selected $\widehat{\mathcal{G}} \subseteq \mathcal{G},|\widehat{\mathcal{G}}|=k$. Since $\widehat{\mathcal{G}}$ is "selected", $z_{i}=1$ for every vertex $v_{i}$ in $\widehat{\mathcal{G}}$ (and $z_{i}=0$ otherwise). Thus $\sum_{i} \sum_{j} z_{i}\left(\gamma_{i j}^{(k-1)}-1\right) z_{j} \neq 0$ implies $\gamma_{i j}^{(k-1)} \neq 1$ for some $v_{i}$ and $v_{j}$ in $\widehat{\mathcal{G}}$. Hence, $\widehat{\mathcal{G}}$ must be disconnected (since there exist $v_{i}$ and $v_{j}$ for which no path of length up to $k-1$ steps joins them).

For the last part of the proposition: (By contradiction.) Suppose (14) holds for some selected connected subgraph $\widetilde{\mathcal{G}} \subseteq \mathcal{G}$, $|\widetilde{\mathcal{G}}|=k$. Assume there exists a selected connected subgraph $\widehat{\mathcal{G}} \subseteq \widetilde{\mathcal{G}},|\widehat{\mathcal{G}}| \leq k$, for which $\sum_{i} \sum_{j} z_{i}\left(\gamma_{i j}^{(k-1)}-1\right) z_{j} \neq 0$. This implies there exists vertices $v_{i}$ and $v_{j}$ in $\widehat{\mathcal{G}}$ such that $\gamma_{i j}^{(k-1)}=0$. But $v_{i}$ and $v_{j}$ are also vertices of $\widetilde{\mathcal{G}}$ for which we know $\gamma_{i j}^{(k-1)}=1$.

Corollary 1. If $k$ is greater than the order of every connected component of $\mathcal{G}$, then (14) does not hold for any selected subgraph of $\mathcal{G}$ with order $k$.

Proof: Let $M$ be the order of the largest connected component of $\mathcal{G}$. Select any $\widehat{\mathcal{G}} \subseteq \mathcal{G}$ such that $|\widehat{\mathcal{G}}|=k$. If $k>M$ then $\widehat{\mathcal{G}}$ must have nonempty intersection with at least two connected components of $\mathcal{G}$. Denote these intersections as $\widehat{\mathcal{G}}^{\prime}$ and $\widehat{\mathcal{G}}^{\prime \prime}$. For each $v_{i}$ in $\widehat{\mathcal{G}}^{\prime}$ and every $v_{j}$ in $\widehat{\mathcal{G}}^{\prime \prime}$ we have $\gamma_{i j}^{(k-1)}=0$ resulting in $z_{i}\left(\gamma_{i j}^{(k-1)}-1\right) z_{j} \neq 0$. Therefore, $\sum_{i} \sum_{j} z_{i}\left(\gamma_{i j}^{(k-1)}-1\right) z_{j} \neq 0$.

Corollary 2. Let $\mathcal{G}_{c}$ be a complete graph-a graph with the property that any two of its vertices are adjacent. Then, for all $k \leq\left|\mathcal{G}_{c}\right|$, any selected subgraph of $\mathcal{G}_{c}$ satisfies (14).

Proof: For every $v_{i}$ and $v_{j}$ in $\mathcal{G}_{c}, \gamma_{i j}^{(k-1)}=1$ for all $k \leq$ $\left|\mathcal{G}_{c}\right|$. Thus, every subgraph of $\mathcal{G}_{c}$ is connected and therefore, by Proposition 2, every selected subgraph satisfies (14).

Example 1. Consider the example shown in Fig. 1. Suppose that, of the four ellipsoids, three are to be selected $(k=3)$ such that the connectedness requirement (9) holds for the selected subset. From the graph representation of the piecewise

\footnotetext{
${ }^{1}$ In (14a), the term 1 is an $N \times N$ matrix with 1 in all its entries.
} 
ellipsoidal set we have

$$
\begin{aligned}
& \Gamma\left(\psi_{k-1}\left(A_{\mathcal{G}}\right)\right)=\Gamma\left(A_{\mathcal{G}}+\left(A_{\mathcal{G}}\right)^{2}\right) \\
& =\Gamma\left(\left[\begin{array}{llll}
1 & 0 & 0 & 1 \\
0 & 1 & 0 & 1 \\
0 & 0 & 1 & 0 \\
1 & 1 & 0 & 1
\end{array}\right]+\left[\begin{array}{llll}
2 & 1 & 0 & 2 \\
1 & 2 & 0 & 2 \\
0 & 0 & 1 & 0 \\
2 & 2 & 0 & 3
\end{array}\right]\right)=\left[\begin{array}{llll}
1 & 1 & 0 & 1 \\
1 & 1 & 0 & 1 \\
0 & 0 & 1 & 0 \\
1 & 1 & 0 & 1
\end{array}\right] .
\end{aligned}
$$

Thus, via Proposition 2, the only possible connected subgraph with order 3 that can be selected consists of the vertex set $\left\{v_{1}, v_{2}, v_{4}\right\}$ corresponding to $\left\{\mathcal{E}_{1}, \mathcal{E}_{2}, \mathcal{E}_{4}\right\}$.

Finally, we note that a simple alternative to the method presented above would be to fix $k$ directions in $\mathcal{J}$ in Lemma 1 and then proceed with computing the maximum volume inscribed ellipsoids corresponding to those directions. However, such a technique (without adequate measures) cannot guarantee that the resulting piecewise ellipsoidal under-approximation 1) is nonempty, 2) is the largest subset among all possible subsets with at most $k$ ellipsoids, and 3 ) is connected.

\section{Application: Control of Anesthesia}

Over the past few years, an interdisciplinary team of researchers at the University of British Columbia has been developing a closed-loop drug delivery system for anesthesia. A bolus-based system has been developed and experimentally validated [22] based on data from more than 80 patients via clinical trials. The long-term goal of this work is to obtain regulatory certificates to fully close the loop, guaranteeing both safety and performance. The viability kernel [9] and the continual reachability set can provide such guarantees.

In [8] we showed that the continual reachability set, as a key step towards closing the loop, can be used to guarantee performance in the control of anesthesia, in that for any initial state in the set, the target clinical effect can be reached at arbitrary times. To compute this set, Laguerre-based LTI models developed previously in [22] describing a patient's dynamic response to rocuronium were used. The state and the input constraint sets $\mathcal{K} \subset \mathbb{R}^{7}$ and $\mathcal{U} \subset \mathbb{R}$ that represent respectively the desired clinical effect and the actuator bounds (hard bounds on the drug infusion rate), are approximated by compact non-degenerate ellipsoids. To compute the continual reachability set $\operatorname{Reach}_{[0, \tau]}^{\gamma}\left(\mathcal{K}_{\downarrow \varepsilon}\right)$, we first computed the maximal reachability sets of the system (with a sampling time of 20s) at every time step in 14 random initial directions for $\tau=90$ time steps. The green/light subset in Fig. 2 shows a projection of a numerically computed under-approximation of the continual reachability set based on (5) and (6). In the $z$ space shown in the figure, the first state $z_{1}$ represents the drug pseudo-occupancy (which is a metric related to the patient's plasma concentration of the anesthetic [22]) minus its setpoint value of 0.9 units, and $z_{2}$ is a Laguerre state transformed from the original coordinate space (cf. [8] for more details).

Based on the computed maximal reachability sets above, we apply Lemma 1 to obtain a piecewise ellipsoidal representation of the continual reachability set (also shown in Fig. 2). For every initial direction $\ell_{\tau} \in \mathcal{J}$, the maximum volume inscribed ellipsoid contained in $\bigcap_{t \in[0, \tau]} \mathcal{E}\left(x^{*}(t), X_{\ell}^{-}(t)\right)$ is

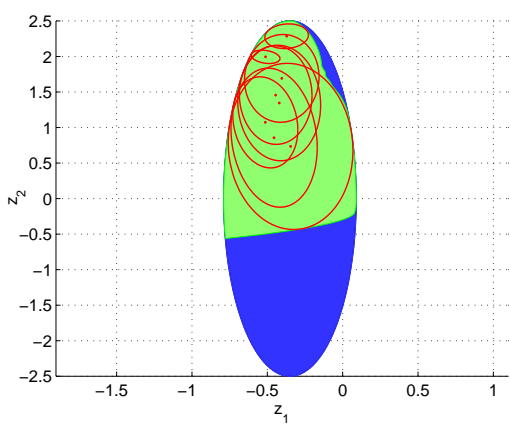

Fig. 2. The $z_{1}-z_{2}$ projection of $\mathcal{K}_{\downarrow \varepsilon}$ (blue/dark) and $\operatorname{Reach} h_{[0, \tau]}^{\gamma}\left(\mathcal{K}_{\downarrow \varepsilon}\right)$ (green/light) computed for patient \#80 undergoing a 30 min surgery $(\tau=90$ with sampling time $\left.T_{s}=20 \mathrm{~s}\right)$. The boundary of a piecewise ellipsoidal representation (red) of $\operatorname{Reach}_{[0, \tau]}^{\gamma}\left(\mathcal{K}_{\downarrow \varepsilon}\right)$ is also shown.

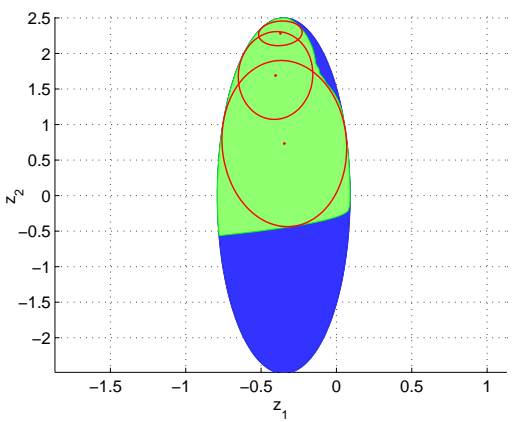

Fig. 3. $z_{1}-z_{2}$ projection of $\operatorname{Reach}_{[0, \tau]}^{\gamma}\left(\mathcal{K}_{\downarrow \varepsilon}\right)$ (green/light) and the boundary of its maximum 3 -connected piecewise ellipsoidal representation (red).

computed using (7). The union of maximum volume inscribed ellipsoids that are nonempty under-approximates the continual reachability set. The resulting piecewise ellipsoidal set consists of 8 distinct (but not necessarily disjoint) nonempty ellipsoids.

To further simplify the representation of $\operatorname{Reach}_{[0, \tau]}^{\gamma}\left(\mathcal{K}_{\downarrow \varepsilon}\right)$, we employ the results of Section III-A and compute a maximum $k$-connected piecewise ellipsoidal representation with $k=3$. To solve the integer program (10) one may use the TOMLAB /CPLEX optimization package [23]. Fig. 3 shows the projection of the simplified representation.

In practice, the representation shown in Fig. 3 provides a set of restrictions that can be straightforwardly implemented, e.g., in conjunction with an MPC-based controller. To ensure safety of the system, the controller is forced to choose an infusion policy that generates a viable trajectory, satisfying the target clinical effect at all times. However, such a policy may not be physiologically suitable for the patient. There are instances in which, depending on the current physiological status of the patient, the anesthesiologist (or the closed-loop controller) may allow the patient to temporarily leave the target in exchange for additional flexibility in selecting a bettersuited (e.g. less aggressive, mildly varying) infusion rate or satisfying other secondary clinical objectives, while ensuring that the patient returns to the target at a prescribed time. The continual reachability set and its associated control laws provide such flexibility. This flexibility stems from the fact 
that, by definition, the viability control laws are a subset of the continual reachability control laws. This will allow the closedloop system to choose an infusion rate that is physiologically more optimized to meet the operating conditions and the patient's ability to handle the anesthetic drug, while simultaneously ensuring that the target clinical effect is reached at a desired time. Such flexible, patient-oriented design has the benefit of tailoring the performance of the system towards the patient's needs during the surgery.

Suppose that the controller/anesthesiologist decides to use a continual reachability control policy, causing the trajectory to temporarily leave the target clinical effect. To guarantee that the trajectory remains in the target once it returns (and therefore guarantee safety), the viability kernel itself can be used as the target set in the computation of the continual reachability set. In that case, $\operatorname{Reach}_{[0, \tau]}^{\gamma}\left(\operatorname{Viab}_{[0, \tau]}(\mathcal{K})\right)=\operatorname{Viab}_{[0, \tau]}(\mathcal{K})$ due to the fact that for $\mathcal{T} \subset \mathcal{X}, \operatorname{Reach}_{[0, \tau]}^{\gamma}(\mathcal{T}) \subseteq \mathcal{T}$. Therefore, $\operatorname{Reach}_{[0, \tau]}^{\gamma}\left(\operatorname{Viab}_{[0, \tau]}(\mathcal{K})\right) \subseteq \operatorname{Viab}_{[0, \tau]}(\mathcal{K})$. On the other hand,

$$
\begin{aligned}
& \forall x \in \operatorname{Viab}_{[0, \tau]}(\mathcal{K}), \exists u(\cdot) \text { s.t. } \forall t, x(t) \in \operatorname{Viab}_{[0, \tau]}(\mathcal{K}) \\
& \Longrightarrow \forall x \in \operatorname{Viab}_{[0, \tau]}(\mathcal{K}), x \in \operatorname{Reach}_{[0, \tau]}^{\gamma}\left(\operatorname{Viab}_{[0, \tau]}(\mathcal{K})\right) \\
& \Longrightarrow \operatorname{Reach}_{[0, \tau]}^{\gamma}\left(\operatorname{Viab}_{[0, \tau]}(\mathcal{K})\right) \supseteq \operatorname{Viab}_{[0, \tau]}(\mathcal{K}) .
\end{aligned}
$$

Thus every state that belongs to the continual reachability set also belongs to the viability kernel, while the viability control laws are still a subset of the continual reachability control laws. In such a scenario, initiating the system using a continual reachability control law allows a temporary relaxation of the state constraint (in favor of a more clinically relevant infusion rate) while ensuring that the patient returns to the new target $\operatorname{Viab}_{[0, \tau]}(\mathcal{K})$ at a prescribed time. Once the trajectory returns to $\operatorname{Viab}_{[0, \tau]}(\mathcal{K})$, the controller will then have the option to apply viability control laws that keep the patient within $\mathcal{K}$. Such mixed scheme has the potential to guarantee not only the performance but also the safety of the closed-loop system.

\section{CONCLUSIONS}

The continual reachability set can facilitate a physiologically more relevant control of anesthesia. The added flexibility in choosing the drug infusion policy provides the anesthesiologist (or the supervisory controller) with additional degrees of freedom to prioritize the patient's needs and the performance of the drug delivery system while still ensuring that the desired clinical effect can be reached at a prescribed time. Motivated by this problem of guaranteed performance in closed-loop control of anesthesia, we presented an underapproximation technique that can be used to compute a simple, fixed-complexity piecewise ellipsoidal representation of the continual reachability set. The proposed closed-form representation paves the way towards, for instance, the synthesis of the continual reachability control laws. Future work also includes practical integration of a safety- and performancebased controller in the closed-loop system. In addition, as what appears to be a straight forward extension of our current results, we also plan to account for the effect of disturbance and uncertainty in the formulation of the continual reachability set and its piecewise ellipsoidal representation. Finally, with an appropriate control synthesis technique in place, it may be interesting to quantify a close estimate of the amount of excursion of a trajectory from the target set based on its initial state and the synthesized continual reachability control laws. (A conservative upper-bound has been provided in [8].)

\section{REFERENCES}

[1] J. Lygeros, "On reachability and minimum cost optimal control," Automatica, vol. 40, no. 6, pp. 917-927, June 2004.

[2] A. Bayen, I. Mitchell, M. Oishi, and C. Tomlin, "Aircraft autolander safety analysis through optimal control-based reach set computation," $J$. Guid. Contr. Dynam., vol. 30, no. 1, pp. 68-77, 2007.

[3] I. Mitchell, A. Bayen, and C. Tomlin, "A time-dependent HamiltonJacobi formulation of reachable sets for continuous dynamic games," IEEE Trans. Auto. Contr., vol. 50, no. 7, pp. 947-957, July 2005.

[4] C. Tomlin, I. Mitchell, A. Bayen, and M. Oishi, "Computational techniques for the verification and control of hybrid systems," Proceedings of the IEEE, vol. 91, no. 7, pp. 986-1001, 2003.

[5] A. Chutinan and B. Krogh, "Computational techniques for hybrid system verification," IEEE Trans. Auto. Contr., vol. 48, pp. 64-75, 2003.

[6] A. B. Kurzhanski and I. Vályi, Ellipsoidal Calculus for Estimation and Control. Boston, MA: Birkhäuser, 1996.

[7] A. N. Daryin, A. B. Kurzhanski, and I. V. Vostrikov, "Reachability approaches and ellipsoidal techniques for closed-loop control of oscillating systems under uncertainty," in Proc. IEEE Conf. Decis. Contr., San Diego, CA, 2006, pp. 6385-6390.

[8] S. Kaynama, M. Oishi, I. Mitchell, and G. Dumont, "The continual reachability set and its computation using maximal reachability techniques," in Proc. IEEE Conf. Decis. Contr., European Contr. Conf., Orlando, FL, Dec. 2011, pp. 6110-6115.

[9] J.-P. Aubin, Viability Theory, ser. Systems and Control: Foundations and Applications. Boston, MA: Birkhäuser, 1991.

[10] O. Simanski, A. Schubert, R. Kaehler, M. Janda, J. Bajorat, R. Hofmockel, and B. Lampe, "Automatic drug delivery in anesthesia: From the beginning until now," in Proc. Mediterranean Conf. Contr. Automation, Athens, Greece, 2007.

[11] C. Ionescu, R. De Keyser, B. Torrico, T. De Smet, M. Struys, and J. Normey-Rico, "Robust predictive control strategy applied for propofol dosing using BIS as a controlled variable during anesthesia," IEEE Trans. Biomed. Eng., vol. 55, no. 9, pp. 2161-2170, 2008.

[12] S. Syafiie, J. Niño, C. Ionescu, and R. De Keyser, "NMPC for propofol drug dosing during anesthesia induction," in Nonlinear Model Predictive Control, ser. Lect. Notes Contr. Inform. Sci. Springer Berlin Heidelberg, 2009, vol. 384, pp. 501-509.

[13] G. Dumont, A. Martinez, and J. Ansermino, "Robust control of depth of anesthesia," Int. J. Adapt. Contr., vol. 23, pp. 435-454, 2009.

[14] P. Oliveira, J. Hespanha, J. Lemos, and T. Mendonça, "Supervised multi-model adaptive control of neuromuscular blockade with off-set compensation," in Proc. European Contr. Conf., 2009.

[15] T. Mendonca, J. A. Lemos, H. Magalhaes, P. Rocha, and S. Esteves, "Drug delivery for neuromuscular blockade with supervised multimodel adaptive control," IEEE Trans. Contr. Syst. Technol., vol. 17, no. 6, pp. 1237-1244, November 2009.

[16] A. B. Kurzhanski and P. Varaiya, "Ellipsoidal techniques for reachability analysis: internal approximation," Syst. Contr. Lett., vol. 41, pp. 201$211,2000$.

[17] A. A. Kurzhanskiy and P. Varaiya, "Ellipsoidal toolbox (ET)," in Proc. IEEE Conf. Decis. Contr., San Diego, CA, Dec. 2006, pp. 1498-1503.

[18] — , "Ellipsoidal techniques for reachability analysis of discrete-time linear systems," IEEE Trans. Auto. Contr., vol. 52, no. 1, pp. 26-38, 2007.

[19] S. Boyd and L. Vandenberghe, Convex Optimization. Cambridge University Press, 2004

[20] R. Cohen and L. Katzir, "The generalized maximum coverage problem," Information Processing Letters, vol. 108, no. 1, pp. 15-22, Sep 2008.

[21] J. Bondy and U. Murty, Graph Theory, ser. Graduate Texts in Mathematics. Springer, New York, 2008, vol. 244.

[22] T. Gilhuly, "Modeling and control of neuromuscular blockade," Ph.D. dissertation, University of British Columbia, Vancouver, Canada, 2007.

[23] K. Holmström, "The TOMLAB optimization environment in Matlab," in Advanced Modeling and Optimization, 1999, pp. 47-69. 